\title{
Hyponatremia is a Predictor of Clinical Outcome for Resected Biliary Tract Cancers: A Retrospective Single-Center Study
}

\author{
Rossana Berardi - Federico Mocchegiani - Silvia Rinaldi · \\ Ilaria Fiordoliva - Francesca Rovinelli · Miriam Caramanti · \\ Alan Federici · Michela Burattini · Francesca Morgese • \\ Mariangela Torniai · Giulia Marcantognini · Marco Vivarelli
}

Received: September 12, 2019 / Published online: April 1, 2020

(C) The Author(s) 2020

\section{ABSTRACT}

Introduction: Biliary tract cancers (BTC) include both gallbladder cancer and cholangiocarcinoma, both of which have a poor prognosis. The aim of our study was to evaluate the main clinical prognostic factors in this setting and to assess their impact on overall survival (OS).

Methods: We retrospectively analyzed data collected on 64 patients with BTC who underwent surgery with radical intent at our institution. OS was estimated using the Kaplan-Meier method. The Cox regression model was used to perform univariate and multivariate analyses.

Results: Preoperative hyponatremia was found to be an independent prognostic factor that

Enhanced Digital Features To view enhanced digital features for this article go to https://doi.org/10.6084/ m9.figshare.11968836.

R. Berardi $(\varangle)$. S. Rinaldi · I. Fiordoliva .

M. Burattini · F. Morgese · M. Torniai .

G. Marcantognini

Clinica Oncologica, Università Politecnica delle

Marche, Università Politecnica delle Marche,

Azienda Ospedaliero-Universitaria Ospedali Riuniti

Umberto I-GM Lancisi-G Salesi, Ancona, Italy

e-mail: r.berardi@univpm.it

F. Mocchegiani · A. Federici · M. Vivarelli Clinica di Chirurgia Epato-biliare, Università Politecnica delle Marche, Azienda OspedalieroUniversitaria Ospedali Riuniti Umberto I-GM Lancisi-G Salesi, Ancona, Italy correlated negatively with prognosis, with hyponatremic patients having a poor OS compared to the group of patients with normal serum sodium levels (9.44 vs. 15.47 months; $p=0.0215$ ). In addition, high preoperative values for carbohydrate antigen 19-9 (Ca19.9), a tumor marker for some gastrointestinal cancers, and lactate dehydrogenase (LDH) were found to be prognostic factors for a significant reduction in OS (Ca19-9: 7.14 vs. 24.22 months, $p=0.0088 ; \quad \mathrm{LDH}: 1.70$ vs. 15.47 months, $p=0.0384$ ).

Conclusion: Identification of these prognostic factors may support strategies to identify, in clinical practice, those subgroups of patients with a favorable or unfavorable prognosis before surgical treatment and, therefore, to guide therapeutic choices. In particular, to our knowledge, this is the first report of the prognostic role of serum sodium level in BTC. Early

\footnotetext{
F. Rovinelli

Department of Medical Oncology, Universita' di Modena e Reggio Emilia, Modena, Italy

M. Caramanti

Clinica Oncologica, Azienda Ospedaliero-

Universitaria Ospedali Riuniti Umberto I, GM

Lancisi, G Salesi, Ancona, Italy

Present Address:

M. Caramanti

UOC Oncologia, Ospedale E.Profili di Fabriano Asur, Marche Area Vasta 2, Ancona, Italy
} 
detection and careful monitoring of hyponatremia and supportive therapy can help to improve the treatment and prognosis of BTC.

Keywords: Biliary tract cancer; Hyponatremia; Prognosis; Surgery

\section{Key Summary Points}

Why carry out this study?

Hyponatremia is an important negative prognostic and predictive factor for cancer patients. However, no data are currently available on the role of hyponatremia in patients with resected biliary tract cancers (BTC).

The aim of this study was to assess the prognostic value of hyponatremia detected at diagnosis in patients with BTC who underwent radical surgery.

\section{What was learned from the study?}

Patients diagnosed with BTC who were also hyponatremic were found to have poorer overall survival than patients with normal preoperative serum sodium levels (9.44 vs. 15.47 months; $p=0.0215$ ).

The results indicate that serum sodium levels should be analyzed in patients with BTC undergoing surgery. Early detection and careful monitoring of hyponatremia and supportive therapy can help to improve patient outcome.

\section{INTRODUCTION}

Biliary tract cancers (BTC) describe a group of gastrointestinal tumors comprising both intraand extra-hepatic cholangiocarcinoma (bile duct cancer) and gallbladder carcinoma. Biliary duct cancer differs from gallbladder cancer in that its incidence is similar in males and females and the survival rate is better [1]. Biliary duct

cancer occurs most frequently in men aged $\geq 70$ years [1].

The symptoms of cholangiocarcinomas occur early, and the disease manifests as a local invasion rather than metastasis. In comparison, gallbladder cancer is more frequent, but it is diagnosed at later stages in most cases since patients are asymptomatic for a long time. The incidence of BTC varies greatly throughout the world [2], with the rate being closely linked with the geographic distribution of risk factors. In Italy, approximately 3000 new cases of BTC are diagnosed each year. BTC represent $0.8 \%$ of all tumors in males and $1.6 \%$ of all tumors in females [3]. However, the incidence of BTC is rising. In particular, the incidence of intrahepatic tumors in the USA rose by $128 \%$ between 1973 and 2012 [4].

Patients with BTC can be asymptomatic or may present jaundice due to the obstruction of the biliary tree caused by the tumor, a palpable mass in the right upper abdomen, abdominal lymphadenopathy, left supraclavicular adenopathy, or fever [5], although only $10 \%$ of patients present with early-stage disease and are eligible for surgical resection, which is the only chance for a cure [6]. Radical surgical resection remains the only curative option, but only $40 \%$ of those who undergo surgery achieve 5-year survival $[7,8]$. Median overall survival (OS) in these patients ranges from 6 to 7 months after surgery alone, with possible prolongation to more than 12 months when a combined approach is used, including chemotherapy either in the adjuvant setting or with disease recurrence [9].

Several prognostic factors before surgery and after resection have been described; of these, jaundice and a high serum level of carbohydrate antigen 19-9 (CA19-9) before surgery are related with a poor prognosis [10]. Hyponatremia has recently been shown to have a negative prognostic impact in cancer patient outcomes. In particular, hyponatremia has been found to be associated with a higher mortality in patients with lung cancer [11], renal cell carcinoma [12], malignant pleural mesothelioma [13], and gastrointestinal cancer [14], while a prompt correction of this electrolyte disorder improved patient outcomes [15]. Hyponatremia has also 
been shown to play a negative predictive role in patients treated with chemotherapy and target therapies [12] and to be associated with a longer hospital stay (and the associated higher costs) [16].

Hyponatremia can be related to diuretic use, gastrointestinal losses, hypotonic infusions, cardiac failure, diabetic impairment, and other causes. In cancer patients, hyponatremia is often caused by the syndrome of inappropriate antidiuretic hormone secretion (SIAD) that results from the ectopic production of antidiuretic hormone. It can also be related to extracellular fluid depletion or renal toxicity related to platinum-based chemotherapy [17]. Its symptoms, especially when the condition is mild or moderate, are non-specific and often lead to a lack of diagnostic classification, resulting in delayed treatment.

To our knowledge, the prognostic impact of hyponatremia in resected BTC patients has not been reported, as we found only two case reports in the literature $[18,19]$. Thus, the aims of our study were to understand the importance of hyponatremia as a prognostic marker both before and after patients with BTC underwent surgery and its impact on outcome and to determine whether the correction of hyponatremia in a timely manner would improve prognosis.

\section{METHODS}

\section{Patient Selection}

We retrospectively analyzed 64 consecutive patients with histologically-confirmed BTC who underwent surgery at our Institution between February 2009 and February 2014 and who were then followed on a regular basis in a specific follow-up program.

\section{Methods}

Patient characteristics and clinical laboratory features that were evaluated include age, sex, symptoms at diagnosis, risk factors and comorbidity, site of primary tumor, type of surgery, blood tests at diagnosis (among others, sodium level, tumor markers (including carcinoembryonic antigen, Ca19-9, and lactate dehydrogenase $[\mathrm{LDH}])$, and OS. Patients received treatment for hyponatremia according to published guidelines [17]. No data on hyponatremia correction were available during the study period. Written informed consent for the diagnostic and therapeutic procedures was obtained from all patients. All procedures performed in the study were in accordance with the ethical standards of the institutional and national research committee and with the 1964 Helsinki declaration and its later amendments or comparable ethical standards. The local ethics committee, Comitato Etico Regione Marche (CERM), approved the study (2020/39, study ID 1315).

\section{Statistical Analysis}

The primary endpoint of this study was to evaluate the prognostic role of hyponatremia before and after surgery for BTC. OS was defined as the interval between radical surgery and death or the last follow-up visit. Patients who were not reported to be deceased at the time of the analysis were censored at the date they were last known to be alive.

The association between categorical variables was estimated using the Chi-square test. The Cox multivariate proportional hazard regression model was used to evaluate the effects of the prognostic factors on survival. Survival distribution was estimated by the Kaplan-Meier method. Significant differences in the probability of surviving between the strata were evaluated by the log-rank test. Hazard ratios and 95\% confidence intervals (CIs) were estimated from regression coefficients. Statistical significance was set at $p \leq 0.05$. All statistical analyses were performed using MedCalc software version 10.4.8 for Windows (MedCalc Software Ltd., Ostend, Belgium).

\section{RESULTS}

Sixty-four patients were included into the study. All patients underwent radical surgery for 
Table 1 Patient characteristics

\begin{tabular}{|c|c|}
\hline Parameter & Values $^{\mathbf{a}}$ \\
\hline Number of patients & 64 \\
\hline Gender (male/female) & $39(60.9 \%) / 25(39.1 \%)$ \\
\hline Median age (years) & $66[46-78]$ \\
\hline \multicolumn{2}{|l|}{ Symptoms at onset } \\
\hline Incidental finding & $19(29.6 \%)$ \\
\hline Jaundice & $15(23.4 \%)$ \\
\hline Abdominal pain & $12(18.7 \%)$ \\
\hline Dyspepsia & $6(9.3 \%)$ \\
\hline Weight loss & $6(9.3 \%)$ \\
\hline Increase in liver function values or indexes of cholestasis & $5(7.8 \%)$ \\
\hline Biliary colic/cholangitis & $5(7.8 \%)$ \\
\hline \multicolumn{2}{|l|}{ Comorbidity/risk factors } \\
\hline Smoke & $10(15.6 \%)$ \\
\hline Biliary calculosis & $4(6.2 \%)$ \\
\hline Previous/attendant cancer in different source & $12(18.75 \%)$ \\
\hline Familiarity for cancers & $17(26.5 \%)$ \\
\hline Hypertension & $31(48.4 \%)$ \\
\hline Diabetes mellitus type 2 & $11(17.1 \%)$ \\
\hline Chronic liver disease (alcohol, hepatitis B and C) & $3(4.6 \%)$ \\
\hline Diverticular disease & $4(6.25 \%)$ \\
\hline \multicolumn{2}{|l|}{ Source of tumor } \\
\hline Intra-hepatic cholangiocarcinoma & $32(50 \%)$ \\
\hline Extra-hepatic cholangiocarcinoma & $15(23.4 \%)$ \\
\hline Gallbladder & $14(21.8 \%)$ \\
\hline Papilla of vater & $3(4.6 \%)$ \\
\hline Death (yes/no) & $45(70.3 \%) / 9(29.6 \%)$ \\
\hline
\end{tabular}

a All values are reported as the number of patients with/without the percentage in parenthesis, with the exception of age, which is presented as the number of years with the range in square bracketsparenthesis

BTC, and no patient received adjuvant therapy. Patient characteristics are summarized in Table 1.

All patients had localized disease with no metastasis, and the histological type was adenocarcinoma in almost all cases. At the time of the statistical evaluation, 45 (70.3\%) patients had died. Median OS after surgery was 16.45 (range 0.13-71.84) months. 
Table 2 Prognostic value of carbohydrate antigen 19-9, lactate dehydrogenase, and hyponatremia prior to surgery

\begin{tabular}{lcr}
\hline Parameter & Number of patients (\%) & Median overall survival (months) \\
\hline CA19-9 elevated before surgery & $2(34.3 \%)$ & 7.14 (range 0.13-20.63) \\
Yes & $25(39.0 \%)$ & 24.22 (range 0.33-58.07) \\
No & $17(26.5 \%)$ & \\
Unknown & $5(7.8 \%)$ & 1.70 (range 0.13-30.79) \\
LDH elevated before surgery & $30(46.8 \%)$ & 15.47 (range 0.33-71.84) \\
Yes & $29(45.3 \%)$ & \\
No & & \\
Unknown & $9(14.06 \%)$ & 9.44 (range 1.70-18.98) \\
Hyponatremia before surgery & $55(85.9 \%)$ & 15.47 (range 0.13-71.84) \\
Yes &
\end{tabular}

CA19-9 Carbohydrate antigen 19-9, LDH lactate dehydrogenase

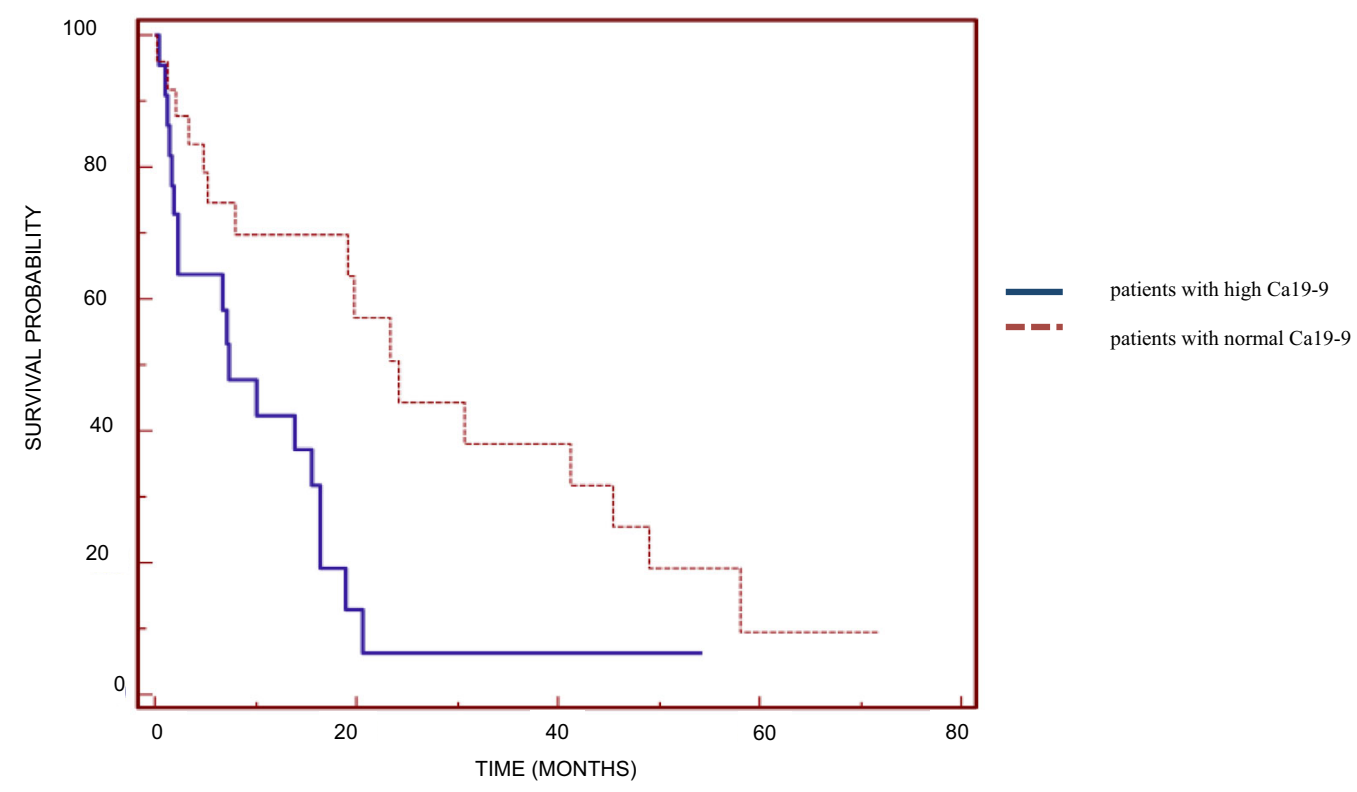

Fig. 1 Kaplan-Meier overall survival curves based on preoperative serum carbohydrate antigen 19-9 (Ca19-9) level for patients with high Ca19-9 levels and those with normal Ca19-9 levels (significant difference at $p=0.0088$ )

Univariate analysis revealed that preoperative values of CA19-9 and LDH and the site of primary tumor were significant prognostic factors (Table 2). Median OS was 7.14 (range 0.13-20.63) months in patients with high values of the tumor marker CA19-9 and 24.22 (range 0.33-58.07) months in those with normal values ( $p=0.0088$; Fig. 1$)$. Median OS was 1.70 (range 0.13-30.79) months in patients with high LDH levels and 15.47 (range 0.33-$71.84)$ months in those with normal levels $(p$ $=0.0384$; Fig. 2 ). With regard to the site of the 


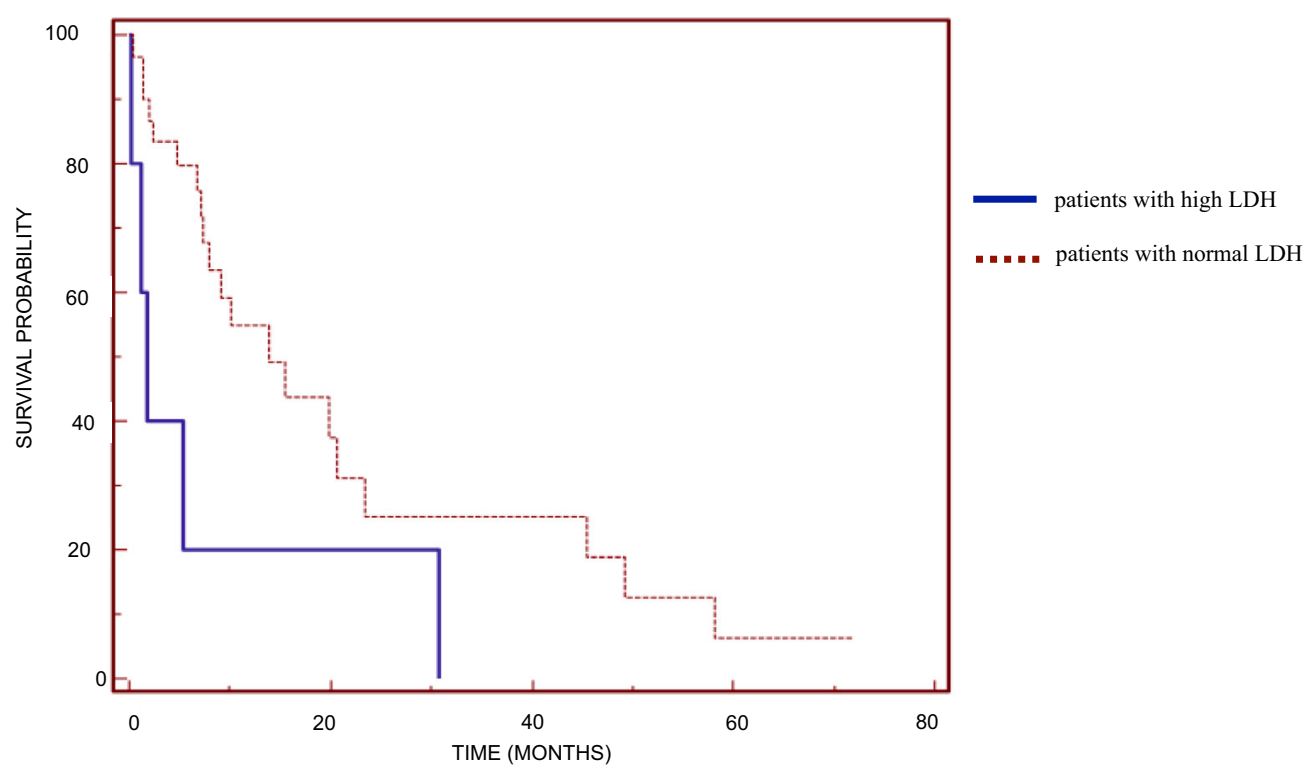

Fig. 2 Kaplan-Meier overall survival curves based on preoperative serum lactate dehydrogenase $(L D H)$ level for patients with high $\mathrm{LDH}$ levels and those with normal LDH levels (significant difference at $p=0.0384$ )

primary tumor, median OS was significantly higher in patients with cholangiocarcinoma (19.8 months, range $1.54-71.84$ months) than in patients with cancer of the gallbladder (7.3 months, range $1.15-41.31$ months) and Klatskin tumor (15.0 months, range 0.33-24.23 months) $(p=0.0422)$.

Univariate analysis also revealed that patients with preoperative hyponatremia (serum sodium $<135 \mathrm{mEq} / \mathrm{L}$ ) had lower survival (median OS 9.44 months, range 1.70-18.98 months) than those with normal serum sodium levels (15.47 months, range 0.13-71.84 months; Fig. 3) ( $p=0.0215)$.

Multivariate analysis of OS, including $\mathrm{LDH}$, CA19-9, and serum sodium levels before surgery, age ( $\geq 65$ vs. $<65$ years), and sex, revealed that only high values of CA19-9 and LDH and hyponatremia before surgery were independent prognostic factors of OS in resected patients with BTC (Table 3).

\section{DISCUSSION}

The results of this study show that preoperative hyponatremia is an independent unfavorable prognostic factor for patients with BTC who undergo radical resection and confirm that preoperative CA19-9 and LDH levels are also independent prognostic factors. These factors may represent simple and useful tools to estimate patient prognosis in this setting.

Regarding CA19-9, a high value of this tumor marker before surgery was found to negatively correlate with survival, similar to the results of our previous study [4] and in agreement with results described in the literature. This result is in agreement with those of Hatzaras et al. who evaluated the prognostic impact of high levels of CA19.9 in a group of 91 patients who underwent radical surgery for cancer of the biliary tract between 1992 and 2007 and found that it was an independent predictor of poor survival [20].

Hyponatremia, on the other hand, has not been previously studied as a prognostic factor for BTC despite it being one of the most common metabolic disorders observed in cancer patients and/or a serious neurological complication, as well as being potentially life-threatening. Hyponatremia is commonly defined as a serum sodium concentration of $<135 \mathrm{mEq} / \mathrm{L}$ [21]. It has been reported to have negative effects on the duration of hospitalization, quality of life, and prognosis, both in cancer 


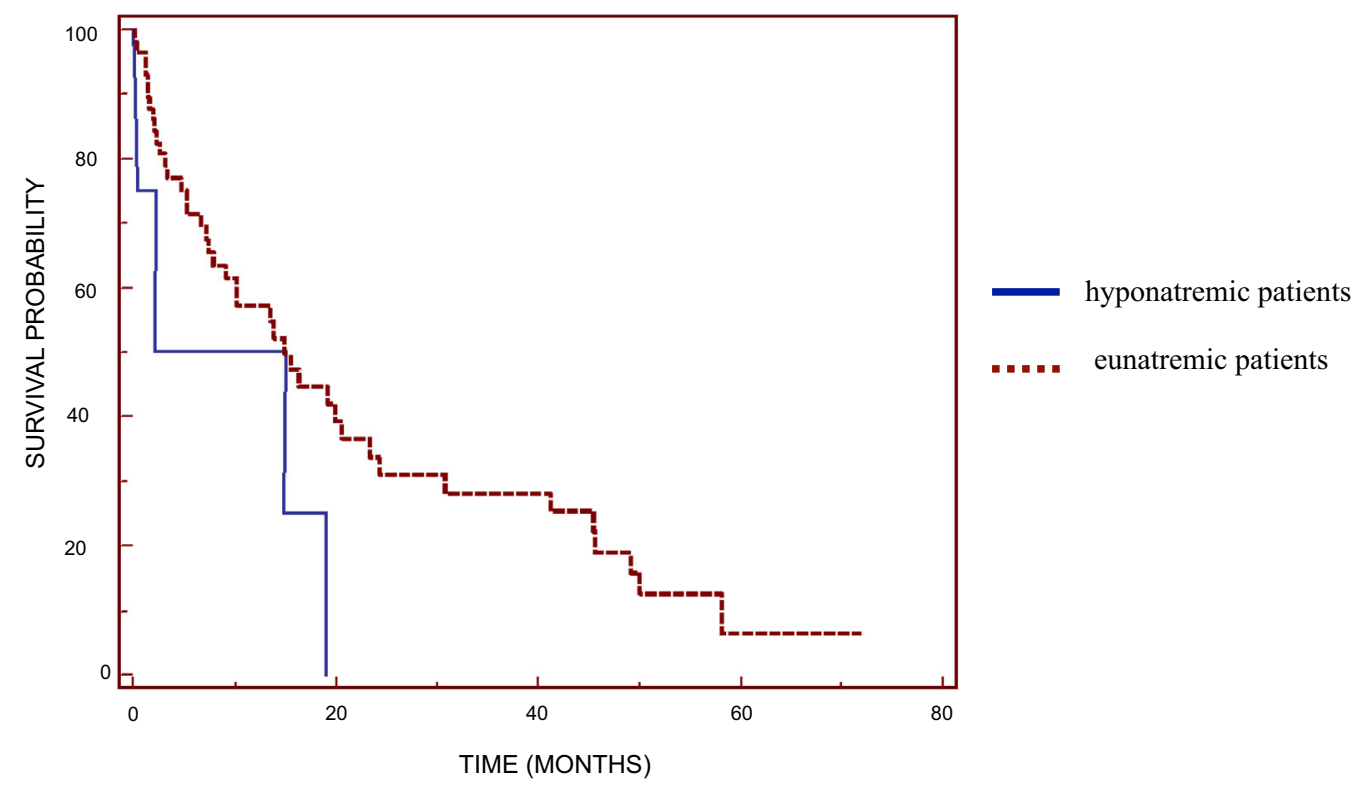

Fig. 3 Kaplan-Meier overall survival curves based on preoperative serum sodium level for hyponatremic and eunatremic patients (significant difference at $p=0.0215$ )

Table 3 Multivariate analysis of overall survival including lactate dehydrogenase, carbohydrate antigen 19-9, and serum sodium levels before surgery, age ( $\geq 65$ vs. $<65$ years), and sex

\begin{tabular}{llll}
\hline Parameter & $\boldsymbol{p}$ & Odds ratio & 95\% Confidence interval \\
\hline LDH & 0.03295 & 0.2861 & $0.0911-0.8983$ \\
Ca19-9 & 0.00147 & 0.1704 & $0.0576-0.5043$ \\
Age $\geq 65$ years & 0.2451 & 1.7006 & $0.6977-4.1450$ \\
Gender & 0.6020 & 0.7697 & $0.2893-2.0480$ \\
Preoperative sodium level & 0.0200 & 0.7697 & $0.5893-0.9480$ \\
\hline
\end{tabular}

patients and in patients with non-neoplastic diseases [16-22].

Treating hyponatremia appears to improve both the symptoms and outcome of the disease, as shown by Waikar et al. who used multivariable-adjusted models to show how patients with hyponatremia had an increased risk of death during hospitalization and at 1 and 5 years after hospitalization, respectively [23]. The increased risk of death was evident even in those with mild hyponatremia (130-134 $\mathrm{mEq} / \mathrm{L})$, and the resolution of hyponatremia during hospitalization attenuated the increased mortality risk conferred by hyponatremia [23].
Hyponatremia is commonly associated with small cell lung cancer (SCLC), as well as with non-small cell lung cancer (NSCLC), lymphomas, gastrointestinal cancers, and mesothelioma [10-15]. Berardi et al. [15] demonstrated that serum sodium normalization is an independent prognostic factor for OS and progression-free survival (PFS) in patients with advanced lung cancer. Patients who achieved normalized serum sodium levels had a higher OS (11.6 vs. 4.7 months, $p=0.0435$ ) and PFS (6.7 vs. 3.3 months, $p=0.011$ ), suggesting the importance of frequent laboratory monitoring and a prompt correction of hyponatremia to 
optimize patient outcome [15]. In another study, patients with NSCLC who developed hyponatremia showed a shorter time to progression to bone lesions (3.73 vs. 5.76 months; $p=0.0187$ ) [24].

Several studies have demonstrated the importance of hyponatremia as a negative prognostic factor in many types of malignant tumors, including lung cancer [25], and hyponatremia has also been studied in breast cancer, tumors of the head and neck, gastrointestinal cancer, gynecologic cancer, acute leukemia, non-Hodgkin's lymphoma and other hematological malignancies. Kim et al. [26] showed that hyponatremia ( $\leq 133 \mathrm{mmol} / \mathrm{L})$ was an important negative prognostic factor for survival in patients with gastric cancer. Similarly, Vasudev et al. [27] showed that it is important to check the serum sodium levels in patients with renal cell carcinoma. These authors studied 212 eunatremic and hyponatremic patients undergoing nephrectomy for renal cell carcinoma and found that survival was increased in all eunatremic patients, persisting even in those in the NOMO subgroup, compared to their hyponatremic counterparts. In eunatremic and hyponatremic patients, the 1-year OS rates were $92.6 \%$ (95\% CI 87.4-97.9) and $77.3 \%$ (95\% CI 69.3-85.3), respectively, and the 5-year OS rates were $67.6 \%(95 \% \mathrm{CI}$ 54.2-80.9) and $44.3 \%$ (95\% CI 32.8-55.8), respectively [27]. In another study, Jappensen et al. [12] identified hyponatremia as a negative prognostic factor in patients with renal cell carcinoma receiving therapy with interleukin-2 and interferon-alpha and that it correlated with performance status, number of involved organs, site of primary tumor, weight loss $>10 \%$, normal leukocytes, low albumin, alkaline phosphatase level above normal, and low hemoglobin level.

Our results show that hyponatremia is a negative prognostic factor in patients with BTC. Although there is a dearth of data on this topic in the literature, the prognostic and predictive role of hyponatremia in other tumors underlines the importance of this factor as a biomarker in patients with BTC as well. Reliable prognostic factors can help the clinician to make the the best therapeutic choice for each patient. In addition, recent evidence shows that correcting the electrolyte disturbance leads to an improvement in the prognosis. Therefore, it is important that the clinician monitor serum sodium levels and correct any related disorders. Confounding factors of hyponatremia, such as use of diuretics, endocrinopathies (e.g., hypothyroidism and hypoadrenalism), and diabetes, must be taken into account in clinical practice [28].

This study has a number of limitations. First, it is a single-center, retrospective study and thus susceptible to bias in terms of data selection and analysis. Second, due to the low incidence of resectable disease, the sample is small; therefore, the results should be validated by future prospective studies. Third, concomitant changes in clinical management and drugs that could not be accounted for in this study can influence hyponatremia.

\section{CONCLUSION}

In conclusion, our results show that hyponatremia can be considered a negative prognostic parameter in resected BTC patients. Therefore, the early detection, monitoring, and management of hyponatremia may improve patient prognosis. In addition, both hyponatremia and Ca19-9 may help to identify subgroups of patients with favorable or unfavorable prognosis prior to surgery and, consequently, to guide further therapeutic choices, eventually leading to the selection of patients who may benefit the most from a postoperative treatment.

To our knowledge this is the first study to evaluate the association of hyponatremia with the outcome of BTC patients. Further investigations are needed to examine the effect of treating hyponatremia on the outcome of these patients.

\section{ACKNOWLEDGEMENTS}

We thank the patients who participated in the study. 
Funding. This research was supported by university funding (Università Politecnica Marche), and the authors did not receive any specific grant from any funding agency in the public, commercial, or not-for-profit sector. No funding or sponsorship was received for the publication of this article.

Authorship. All named authors meet the International Committee of Medical Journal Editors (ICMJE) criteria for authorship for this article, take responsibility for the integrity of the work as a whole, and have given their approval for this version to be published.

Disclosures. Rossana Berardi received a consulting fee from Otsuka and is an Editor-inChief for Oncology and Therapy. Federico Mocchegiani, Silvia Rinaldi, Ilaria Fiordoliva, Francesca Rovinelli, Miriam Caramanti, Alan Federici, Michela Burattini, Francesca Morgese, Mariangela Torniai, Giulia Marcantognini, Marco Vivarelli have nothing to disclose.

Compliance with Ethics Guidelines. Written informed consent for the diagnostic and therapeutic procedures was obtained from all patients. All procedures performed in the study were in accordance with the ethical standards of the institutional and national research committee and with the 1964 Helsinki declaration and its later amendments or comparable ethical standards. The local ethics committee, Comitato Etico Regione Marche (CERM), approved the study (2020/39, study ID 1315).

Data Availability. The datasets analyzed during the current study are available from the corresponding author on reasonable request.

Open Access. This article is licensed under a Creative Commons Attribution-NonCommercial 4.0 International License, which permits any non-commercial use, sharing, adaptation, distribution and reproduction in any medium or format, as long as you give appropriate credit to the original author(s) and the source, provide a link to the Creative Commons licence, and indicate if changes were made. The images or other third party material in this article are included in the article's Creative Commons licence, unless indicated otherwise in a credit line to the material. If material is not included in the article's Creative Commons licence and your intended use is not permitted by statutory regulation or exceeds the permitted use, you will need to obtain permission directly from the copyright holder. To view a copy of this licence, visit http://creativecommons.org/licenses/by$\mathrm{nc} / 4.0 /$.

\section{REFERENCES}

1. Bragazzi MC, Cardinale V, Carpino G, et al. Cholangiocarcinoma: epidemiology and risk factors. Transl Gastrointest Cancer. 2012;1:21-32.

2. Shaib Y, El-Serag HB. The epidemiology of cholangiocarcinoma. Semin Liver Dis. 2004;24(2):115-25.

3. Patel N, Benipal B. Incidence of cholangiocarcinoma in the USA from 2001 to 2015: A US cancer statistics analysis of 50 states. Cureus. 2019;11(1): e3962.

4. Siegel R, Ma J, Zou Z, Jemal A. Cancer statistics, 2014. CA Cancer J Clin. 2014;64(1):9-29.

5. Berardi R, Mocchegiani F, Pierantoni C, et al. Resected biliary tract cancers: a novel clinicalpathological score correlates with global outcome. Dig Liver Dis. 2013;45(1):70-4.

6. Hezel AF, Zhu AX. Systemic therapy for biliary tract cancers. Oncologist. 2008;13:415-23.

7. Yeo CJ, Cameron JL, Sohn TA, et al. Six hundred fifty consecutive pancreaticoduodenectomies in the 1990s: pathology, complications and outcomes. Ann Surg. 1997;226(3):248-57.

8. Benson AB 3rd, D'Angelica MI, Abbott DE, et al. NCCN guidelines insights: hepatobiliary cancers, version 1.2017. J Natl Compr Cancer Netw. $2014 ; 15(5): 563-73$.

9. Krasnick BA, Jin LX, Davidson JT 4th, et al. Adjuvant therapy is associated with improved survival after curative resection for hilar cholangiocarcinoma: a multi-institution analysis from the US extrahepatic biliary malignancy consortium. J Surg Oncol. 2018;117(3):363-71.

10. Wellner UF, Shen Y, Keck T, Jin W, Xu Z. The survival outcome and prognostic factors for distal cholangiocarcinoma following surgical resection: a 
meta-analysis for the 5-year survival. Surg Today. 2017;47(3):271-9.

11. Hansen O, Sørensen P, Hansen KH. The occurrence of hyponatremia in SCLC and the influence on prognosis: a retrospective study of 453 patients treated in a single institution in a 10 -year period. Lung Cancer. 2010;68:111-4.

12. Jeppesen AN, Jensen HK, Donskov F, Marcussen N, von der Maase H. Hyponatremia as a prognostic and predictive factor in metastatic renal cell carcinoma. Br J Cancer. 2010;102:867-72.

13. Berardi R, Caramanti M, Fiordoliva $I$, et al. Hyponatraemia is a predictor of clinical outcome for malignant pleural mesothelioma. Support Care Cancer. 2015;23(3):621-6.

14. Choi JS, Bae EH, Ma SK, Kweon SS, Kim SW. Prognostic impact of hyponatraemia in patients with colorectal cancer. Colorectal Dis. 2015;17:409-16.

15. Berardi R, Santoni M, Newsom-Davis T, et al. Hyponatremia normalization as an independent prognostic factor in patients with advanced nonsmall cell lung cancer treated with first-line therapy. Oncotarget. 2017;8:23871-9.

16. Berardi R, Caramanti $M$, Castagnani $M$, et al. Hyponatremia is a predictor of hospital length and cost of stay and outcome in cancer patients. Support Care Cancer. 2015;23:3095-101.

17. Castillo JJ, Vincent M, Justice E. Diagnosis and management of hyponatremia in cancer patients. Oncologist. 2012;17(6):756-65.

18. Ng ES, Venkateswaran K, Ganpathi SI, Chuah BY. Small cell gallbladder carcinoma complicated by paraneoplastic hyponatremia: a case report and literature review. J Gastrointest Cancer. 2010;41(4): 264-8.

19. Saleem A. Severe hyponatremia presenting as paraneoplastic syndrome in a patient with small cell carcinoma of gallbladder. J Coll Physicians Surg Pak. 2016;26(5):451-2.

20. Hatzaras I, Schmidt C, Muscarella P, Melvin WS, Ellison EC, Bloomston M. Elevated CA 19-9 portends poor prognosis in patients undergoing resection of biliary malignancies. HPB (Oxford). 2010;12(2):134-8.

21. Adrogué HJ, Madias NE. Hyponatremia. N Engl J Med. 2000;342(21):1581-9.

22. Doshi SM, Shah P, Lei X, Lahoti A, Salahudeen AK. Hyponatremia in hospitalized cancer patients and its impact on clinical outcomes. Am J Kidney Dis. 2012;59(2):222-8.

23. Waikar SS, Mount DB, Curhan GC. Mortality after hospitalization with mild, moderate, and severe hyponatremia. Am J Med. 2009;122(9):857-65.

24. Rinaldi S, Santoni M, Leoni G, et al. The prognostic and predictive role of hyponatremia in patients with advanced non-small cell lung cancer (NSCLC) with bone metastases. Support Care Cancer. 2019;27(4):1255-61.

25. Zarzecka M, Kubicki P, Kozielski J. Hyponatraemia-evaluation of prevalence in patients hospitalized in the pulmonary department and prognostic significance in lung cancer patients. Pneumonol Alergol Pol. 2014;82(1):18-24.

26. Kim HS, Yi SY, Jun HJ, et al. Clinical outcome of gastric cancer patients with bone marrow metastases. Oncology. 2007;73:192-7.

27. Vasudev NS, Brown JE, Brown SR, et al. Prognostic factors in renal cell carcinoma: association of preoperative sodium concentration with survival. Clin Cancer Res. 2008;14(6):1775-81.

28. Berardi R, Rinaldi S, Caramanti M, et al. Hyponatremia in cancer patients: time for a new approach. Crit Rev Oncol Hematol. 2016;102:15-25. 